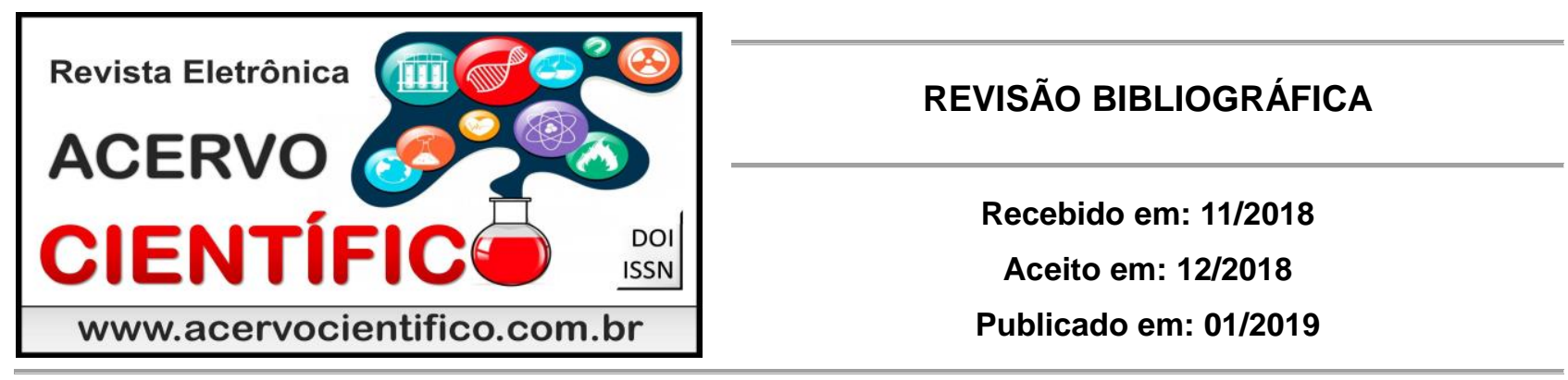

\title{
Uma revisão bibliográfica sobre aterosclerose
}

\author{
A literature review on atherosclerosis \\ Una revisión bibliográfica sobre aterosclerosis
}

\section{Samuel Cavalcante Reis ${ }^{1}$}

\begin{abstract}
Resumo: O objetivo desse estudo foi reconhecer por meio de uma revisão da literatura científica a fisiopatologia da aterosclerose, bem como suas possíveis causas, tratamentos e seus problemas relacionados. Sendo assim, propôs-se um estudo descritivo de revisão bibliográfica, realizada por meio de base de dados de artigos da literatura cientifica. Tendo-se em vista todas as conseqüências da aterosclerose, estão sendo enviados esforços intensivos para desenvolver meios de redução dos índices de incidência, já que esses meios se deparam diretamente envolvidos com os programas de prevenção primária, cujo objetivo é retardar a formação do ateroma ou induzir regressão das lesões.
\end{abstract}

Palavra-chave: Aterosclerose; causas; tratamentos; problemas relacionados.

\begin{abstract}
The aim of this study was to recognize, through a review of the scientific literature, the pathophysiology of atherosclerosis, as well as its possible causes, treatments and related problems. Thus, a descriptive study of bibliographic review was proposed, based on a database of articles in the scientific literature. In view of all the consequences of atherosclerosis, intensive efforts are being made to develop means of reducing incidence rates, since these are directly involved with primary prevention programs, which aim to delay atheroma formation or induce regression of the lesions.
\end{abstract}

Keywords: Atherosclerosis; causes; treatments; problems.

Resumen: El objetivo de este estudio fue reconocer por medio de una revisión de la literatura científica la fisiopatología de la aterosclerosis, así como sus posibles causas, tratamientos y sus problemas relacionados. Siendo así, se propuso un estudio descriptivo de revisión bibliográfica, realizada por medio de base de datos de artículos de la literatura científica. En vista de todas las consecuencias de la aterosclerosis, se están realizando esfuerzos intensivos para desarrollar medios de reducción de los índices de incidencia, ya que estos medios se enfrentan directamente involucrados con los programas de prevención primaria, cuyo objetivo es retrasar la formación del ateroma o inducir la regresión de las lesiones.

Palabra clave: Aterosclerosis; causa; tratamientos; problemas relacionados.

${ }^{1}$ Graduado em Medicina pela Faculdades Integradas Aparício Carvalho (FIMCA), Porto Velho-RO. E-mail: samuel 2761@hotmail.com 


\section{INTRODUÇÃO}

Dados de literatura científica mostram um conseso sobre o estádio mais precoce da aterogênese as quais é caracterizado pelo acúmulo focal de macrófagos com deposição lipoproteínas de baixa densidade (Low Density Lipoproteins, LDL) e colesterol na camada íntima arterial. Em outras palavras, ocorre alterações morfológicas por causa do acúmulo de célula espumosa no espaço subendotelial do vaso, o que caracteriza o estágio mais precoce da aterosclerose (SUMIDA K, et al., 2018).

Certamente, grupos de pesquisa de todo o mundo tentam caracterizar e investigar os mecanismos moleculares que precedem a formação das células espumosas na tentativa de estabelecer métodos preventivos precosses que possam interferir na formação e na evolução da placa de aterosclerose (BIJNEN M, et al., 2018).

Até o presente momento a explicação científica comum a nível molecular, caracterizadas por modelos experimentais in vivo e in vitro, observou-se que as alterações da aterosclerose são decorrentes do carreamento de LDL plasmático, que é capaz de permear e se depor na região da íntima arterial, e por conseguinte é oxidado em consequência do acumulo de radicais livres que são gerados nessa região. Essa oxidação gera um estresse local e induz a células musculares lisas da camada intima a produzirem substâncias quimioatractantes que são capazes de induziriam a migração de monócitos, que se deslocam do plasma para dentro da camada íntima e se diferenciam em macrofagos ativados capazes de fagocitar 0 LDL e consequentemente o corre a formação das células espumosas (FRANZAGO M, et al., 2018; BIJNEN $\mathrm{M}$, et al., 2018).

De modo geral, a explicação acima propoem um mecanismo que explica o desenvolvimento das células espumosas, entre tanto, não discute como as LDL plasmáticas atingem a região intimal do vaso e tão pouco quais são as causas decorrentes desse processo, além das alterações metabólicas já conhecidas. Este é um ponto ainda não bem esclarecido entre os estudiosos da aréa, mas cuja discussão tem a maior importância na tentativa de intervenções precosses que possam prevenir o desenvolvimento da doença (DE WINTHER MPJ, DALLINGA-THIE GM, 2018; FRANZAGO M, et al., 2018).

Diante disso, propôs-se um estudo descritivo de revisão bibliográfica, realizada por meio de base de dados de artigos da literatura cientifica a fim de reconhecer por meio de uma revisão da literatura científica a fisiopatologia da aterosclerose, bem como suas possíveis causas, tratamentos e seus problemas relacionados.

\section{RESULTADOS E DISCUSSÃO}

A aterosclerose resumidamente e esporadicamente significa o endurecimento das artérias; mais corretamente, entretanto, trata-se de um termo genérico para referir-se a três padrões de doenças vasculares que possuindo em comum o espessamento e perda da elasticidade das paredes arteriais (SANTOS RD, MARANHÃO RC, 1998). A aterosclerose caracteriza-se por lesão da camada íntima, denominada ateromas ou placa fibrogordurosa, que fazem protrusão na luz, enfraquecem a média subjacente e sofrem uma série de complicações. A aterosclerose contribui esmagadoramente para uma taxa de mortalidade - cerca da metade ou mais de todas as mortes - e morbidade, no mundo ocidental, que ultrapassa a de qualquer outra doença. Sendo de distribuição mundial, atingindo proporções epidemiológicas nas sociedades economicamente desenvolvidas (GARCIA YM, et al., 2000; SUMIDA K, et al., 2018).

O padrão dominante é a aterosclerose, caracterizada pela formação de placas fibrosas na íntima, que quase sempre exibem um centro grumoso ríco em lipídio (SANTOS RD, et al., 2000; BIJNEN M, et al., 2018).

A segunda forma morfológica de aterosclerose, de importância clínica bem menor, é a esclerose medial calcificada de Mönckeberg, caracterizada por depósitos calcificados na artérias musculares de calibre médio de indivíduos com idade maior que 50 anos. As calcificações muitas vezes sofrem ossificações assumem a 
forma de placas mediais irregulares ou anéis transverso isolado; criam uma nodularidade à palpação e são facilmente visualizadas em radiografia. Apesar de estar lesões mediais não invadirem a luz do vaso, as artérias afetadas também podem desenvolver aterosclerose (ZOFFI RS, GERBER PZ, 1997; FRANZAGO $\mathrm{M}$, et al., 2018).

O terceiro padrão consiste em doença das pequenas artérias e arteríolas (arteriolosclerose). As duas variantes anatômicas - hialina e hipeplásica - causam espessamento das paredes vasculares com estreitamento da luz, podendo induzir lesão isquêmica. A arteriolosclerose está, mais freqüentemente associada com hipertensão e diabetes mellitus (BATHOUNI M, 1997; SUMIDA K, et al., 2018).

Zoffi RS e Gerber PZ descreveram a patogenia da dença e propuzeram as características apresentadas no Quadro 1, abaixo:

Quadro 1 - Patogenia da aterosclerose seguindo Zoffi RS (1997) e SUMIDA K, et al. (2018).

* Desenvolvimento de regiões focais de lesão endotelial crônica, geralmente sutil, com conseqüente disfunção endotelial, como aumento da permeabilidade endotelial e aumento da adesão dos leucócitos.

* Acúmulo de lipoproteínas na parede do vaso, principalmente LDL com seu elevado conteúdo de colesterol, bem como de lipoproteínas de densidade muito baixa (VLDL), e modificação destas lipoproteínas por oxidação.

* Adesão dos monócitos sangüíneos (e outros leucócitos) ao endotélio, seguido de migração destas células na íntima e sua transformação em macrófagos e células espumosas.

* Adesão de plaquetas as áreas focais de desnudação (quando presentes) ou a leucócitos aderente.

* Liberação de fator das plaquetas ativadas, macrófagos ou células vasculares, que induzem a migração das células musculares lisas da média para a íntima.

- Proliferação das células musculares lisas da íntima e elaboração de matriz extracelular, resultante em acúmulo de colágeno e de proteoglicanos.

* Aumento do acúmulo de lipídios tanto no interior das células (macrófagos e células musculares lisas) quanto no meio extracelular.

Fonte: Quadro confeccionado a partir de Zoffi RS e Gerber PZ (1997); SUMIDA K, et al. (2018).

A respeito do contínuo impacto da doença relacionada com a aterosclerose sobre a saúde, foram efetuados notáveis progresso, durante as últimas décadas, nos Estados Unidos e em outros países. Entre o anos de 1963 (o ano de maior incidência) e 1995, houve uma redução de cerca de 50\% na taxa de mortalidade por acidentes vasculares encefalicos - uma redução que aumentou em cinco anos a redução a perspectivas de vida do indivíduo nos EUA. Esta tendência tem sido atribuida à prevenção da aterosclerose através da mudança do estilo de vida, incluindo redução do fumo de cigarros, alterações dos hábitos com consumo reduzido de colesterol e outras gorduras animal saturadas e controle da hipertensão; e uma melhora no tratamento do infarto do miocárdio e outras complicações da cardiopatia isquêmica (SANTOS RD, et al., 2000; SUMIDA K, et al., 2018).

A aterosclerose afeta primariamente as artérias elásticas (por exemplo, aorta, artérias carótidas e ilíacas) e as artérias musculares de grande e médio calibre (por exemplo, artéria coronária e poplíteas). A doença quase sempre começa na infância; entretanto, os sintomas habitualmente só se tornam evidente na meiaidade ou mais tarde, quando as lesões arteriais precipitam lesão orgânica (SANTOS RD, MARANHÃO RC, 1998; BIJNEN M, et al., 2018). 
Embora qualquer orgão ou tecido do corpo possa ser afetado, a doença ateroscleróticas sintomática localiza-se mais freqüente nas artérias que suprem o coração, o cérebro, os rins, os membros inferiores e o intestino delgado. As principais conseqüência desta doença consiste em infarto do miocárdio (ataque cardíaco), infarto cerebral (acidente vascular encefálico) e aneurisma da aorta. Por conseguinte, os dados epidemiológicos da aterosclerose são expressos em grande parte em termos da incidência ou do número de morte causadas por cardiopatias isquêmicas. A aterosclerose também é responsável por outras consequências da redução aguda ou crônica da perfusão arterial, como gangrena das pernas, oclusão mesentérica, morte cardiopática, súbita, cardiopatia isquémica crônica e encefalite isquêmica (BATHOUNI M, 1997; DE WINTHER MPJ, DALLINGA-THIE GM, 2018).

Os ateromas a princípio exibem distribuição focal e esparsa, porém tornam-se mais numerosos à medida que a doença evolui, chegando, algumas vezes, a cobrir toda a circunferência das artérias gravemente afetadas Por conseqüência, nas pequenas artérias, os ateromas são oclusivos, comprometendo o fluxo sangüíneo para órgãos distantes e causando lesão isquêmica. Além disso as placas podem sofrer ruptura e precipitar trombos que obstruem ainda mais o fluxo sangüíneo (GARCIA YM, et al., 2000).

Nas artérias de grande calibre, são destrutivas, invadem a média subjacente e enfraquecem a parede vascular afetada, causando aneurisma ou ruptura, ou favorecendo o desenvolvimento de trombose. Além disso, os ateromas externos são friáveis, liberando freqüentemente êmbolos na circulação distal suprido pela aorta descendente e ascendente (ZOFFI RS, GERBER PZ, 1997; FRANZAGO M, et al., 2018).

Os processos básicos na aterosclerose consistem em espessamento e acúmulo de lipídios na íntima, produzindo as placas de ateroma características. Como principal causa de estreitamento arterial em adultos, estas lesões são descritas em primeiro lugar (SANTOS RD, et al., 2000).

A lesão básica consiste numa placa focal elevada no interior da íntima, com centro de lipídio (principalmente colesterol e ésteres de colesterol) e uma cápsula fibrosa. Exibem coloração branca a branco-amarelada e projetam-se na luz da artéria. Seu tamanho varia de 0,3 a $1,5 \mathrm{~cm}$ de diâmetro: entretanto, as placas algumas vezes coalescem, formando massas maiores (JORGE PAR, 1997; FRANZAGO M, et al., 2018).

Em cortes, a porção superficial desta lesão na superfície luminal tende a ser de consistência firme e banca (cápsula fibrosa), enquanto as porções profundas são amareladas ou amarelo-esbranquiçadas e moles. O centro das placas maiores pode conter um resíduo grumoso amarelado, daí o termo ateroma, um nome alternativo para referir-se a placa aterosclerótica, derivada da palavra grega para mingau (BATHOUNI M, 1997; TANG LH, et al., 2018).

A distribuição das placas ateroscleróticas nos seres humanos e característica. A aorta abdominal geralmente é muito mais afetada a aorta torácica, e as lesões aórticas tendem a ser muito mais proeminente em torno da origem (óstios) de seus principais ramos (SANTOS RD e autores, 2000; KOENIG W, 2018).

As placas de aterosclerótica possuem três componentes principais: (1) células, incluindo células musculares lisas, macrófagos, e outros leucócitos; (2) matriz extracelular do tecido conjuntivo, incluindo colágeno, fibras elásticas e proteoglicanos; e (3) depósito lipídicos intracelulares e extracelulares (KOENIG W, 2017).

Tipicamente, a cápsula fibrosa superficial é composta de células musculares lisas, com poucos leucócitos e tecido conjuntivo relativamente denso; de uma área celular abaixo a ao lado da cápsula, que consiste numa mistura de macrófagos, células musculares lisas e linfócitos T; e de um centro necrótico mais profundo no qual se encontra uma massa disforme de material lipídico, fenda de colesterol, restos celulares, células espumosas repletas de lipídio, fibrina e outra proteínas plasmáticas (SANTOS RD, MARANHÃO RC, 1998; CHRISTEN T, et al., 2018). 
As células espumosas derivam predominantemente dos monócitos sangüineo (que se transformam em macrófagos); entretanto, as células musculares lisas também podem fagocitar lipídios, transformando-se em células espumosas (GARCIA YM, et al., 2000; DE WINTHER MPJ, DALLINGA-THIE GM, 2018).

Os fatores de risco são: idade mais avançadas, sexo masculino, predisposição familia, hiperlipidemia (LDL e VLDL), hipertensão, tabagismo , diabetes mellitus e fator múltiplo (PECOITS-FILHO R, et al., 2002).

A prevenção primária se dá através de modificação dos fatores de risco: abstenção ou interrupção do fumo de cigarros,; controle da hipertensão; controle do diabetes mellitus, controle da hipocolesterolemia, redução do peso através de controle da ingestão calórica total, juntamente com aumento dos exercícios; moderação no consumo de álcool; e mais importante ainda, a redução dos níveis sangüíneos totais de colesterol e de colesterol das LDL, enquanto se aumenta a concentração das HLDL (BATHOUNI M, 1997; SUMIDA K, et al., 2018).

\section{CONSIDERAÇÕS FINAIS}

Tendo-se em vista todas as conseqüências da aterosclerose, estão sendo enviados esforços intensivos para desenvolver meios de redução dos índices de incidência, já que esses meios se deparam diretamente envolvidos com os programas de prevenção primária, cujo objetivo é retardar a formação do ateroma ou induzir regressão das lesões.

Sendo assim este estudos nos proporcionou maior conhecimento sobre a aterosclerose, bem como toda informação necessárias para e realizações de ações práticas preventivas ou redutivas ao portador de aterosclerose.

\section{REFERÊNCIAS}

1. BATHOUNI M. Hipótese Oxidativa da Aterosclerose e Emprego dos Antioxidantes na Doença Arterial Coronária. Arq Bras Cardiol volume 68, (no 1), 1997.

2. SUMIDA K, et al., et al. Constipation and risk of death and cardiovascular events. Atherosclerosis. 2018; 281:114-120. doi: 10.1016/j.atherosclerosis.2018.12.021.

3. FRANZAGO M, et al. Early Subclinical Atherosclerosis in Gestational Diabetes: The Predictive Role of Routine Biomarkers and Nutrigenetic Variants. J Diabetes Res. 2018, 24;2018:9242579. doi: 10.1155/2018/9242579.

4. GARCIA YM, et al. Fatores de Risco para Aterosclerose em uma População Idosa Ambulatorial na Cidade de São Paulo. Arq Bras Cardiol, volume 74 (nํ 3), 181-188, 2000.

5. JORGE PAR. Endotélio, lipídios e Aterosclerose. Arq. Bras. Cardiol volume 68, (no 2), 1997.

6. KUMAR, Vinay; COTRAN, Ramzi S; ROBBINS, Stanley L. Patologia Básica. 5ํed. Edt. Guanabara Koogan S.A. Rio de Janeiro, 1994. 322-324.

7. DE WINTHER MPJ, DALLINGA-THIE GM. Introduction to the thematic review series on different levels of genetic regulation of cardiovascular disease. Atherosclerosis. 2018. pii: S00219150(18)31545-4.

8. BIJNEN M, et al. Adipose tissue macrophages do not affect atherosclerosis development in mice. Atherosclerosis. 2018;281:31-37. doi: 10.1016/j.atherosclerosis.2018.12.010.

9. PECOITS-FILHO R, et al. Revisão: Desnutrição, inflamação e aterosclerose (síndrome MIA) em pacientes portadores de insuficiência renal crônica. J Bras Nefrol 2002;24(3):136-46. 
10. ROBINS Atanley L; KUMAR, Vinay; COTRAN, Ramzi. Patologia, 6e ed. Edt. Guanabara Koogan S.A. Rio de Janeiro, 2000. 639-640.

11. ROMALDINI CC, et al. Fatores de risco para aterosclerose em crianças e adolescentes com história familiar de doença arterial coronariana prematura. Jornal de Pediatria - Vol. 80, №2, 2004.

12. SANTOS RD, et al. I DIRETRIZ DE PREVENÇÃO DA ATEROSCLEROSE NA INFÂNCIA E NA ADOLESCÊNCIA. Arquivos Brasileiros de Cardiologia - Volume 85, Suplemento VI, Dezembro 2005.

13. SANTOS RD, et al. III Diretrizes Brasileiras Sobre Dislipidemias e Diretriz de Prevenção da Aterosclerose do Departamento de Aterosclerose da Sociedade Brasileira de Cardiologia. Arq Bras Cardiol volume 77, (suplemento III), 2001.

14. SANTOS RD, et al. Programa de Avaliação Nacional do Conhecimento sobre Prevenção da Aterosclerose (PANDORA). Um Questionário entre Cardiologistas Brasileiros Sobre Redução do Colesterol. Arq Bras Cardiol, volume 75 ( $n^{\circ}$ 6), 289-295, 2000.

15. SANTOS RD, MARANHÃO RC. Comparação Entre Homens E Mulheres Hipercolesterolêmicos De Alto Risco De Desenvolvimento De Aterosclerose. Estudo Dos Fatores De Risco E Da Resposta Ao Tratamento Com Pravastatina. Arq. Bras Cardiol, volume 70 (ํㅜ 6), 383-387, 1998.

16. STEVENS, Alan; LOWE, James. Patologia. º ed. Edt. Manole. São Paulo, 2002. 128-132.

17. ZOFFI RS, GERBER PZ. Fatores de Risco de Aterosclerose na Infância. Um Estudo Epidemiológico. Arq Bras Cardiol, volume 69 (nํ4), 231-236, 1997.

18. KOENIG W. Inflammation Revisited: Atherosclerosis in The Post-CANTOS Era. Eur Cardiol. 2017 Dec;12(2):89-91. doi: 10.15420/ecr.2017:18:1.

19. TANG LH, HUANG C, FENG YQ. Serum total bilirubin concentration is associated with carotid atherosclerosis in patients with prehypertension. Clin Exp Hypertens. 2018 Oct 30:1-5. doi: 10.1080/10641963.2018.1539094.

20. CHRISTEN T, et al. Mendelian randomization analysis of cholesteryl ester transfer protein and subclinical atherosclerosis: A population-based study. J Clin Lipidol. 2018 Jan - Feb;12(1):137144.e1. doi: 10.1016/j.jacl.2017.10.023. 